\title{
Exploring the LINC to nuclear envelope spacing
}

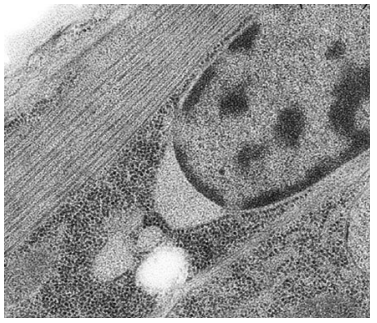

Electron microscopy shows a large gap between the inner and outer nuclear membranes of a worm muscle cell lacking UNC-84.

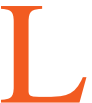

INC complexes do not maintain the space between nuclear membranes, except in cells exposed to mechanical stress, Cain et al. reveal.

SUN proteins in the inner nuclear membrane and $\mathrm{KASH}$ proteins in the outer nuclear membrane form LINC complexes that regulate multiple processes by connecting the nucleoskeleton to the cytoskeleton. LINC complexes are also thought to maintain a uniform gap of $\sim 50 \mathrm{~nm}$ between the outer and inner nuclear membranes. This perinuclear space widens in HeLa cells depleted of SUN proteins, but the role of LINC complexes in maintaining nuclear envelope architecture in vivo hasn't been tested.

Cain et al. examined the nuclear envelopes of $C$. elegans

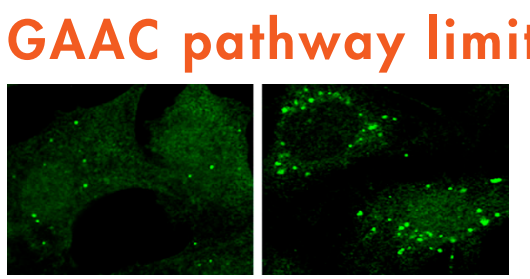

Cells lacking ATF4 (right) contain more autophagosomes (green) than control cells (left) after 12 hours of mild starvation. larvae lacking the only somatic SUN protein, UNC-84. Surprisingly, the absence of UNC-84 had no effect on nuclear envelope spacing in most worm tissues. In the body wall muscle cells of unc-84 mutants, however, the nuclear membranes became widely separated in regions predicted to undergo mechanical strain. Cain et al. therefore think that LINC complexes are only required to hold nuclear membranes together in cells subjected to high mechanical stress. Consistent with this, HeLa cells experience intracellular tension when plated onto tissue culture dishes.

Cain et al. wondered whether the size of LINC complexes nevertheless sets the width of the perinuclear space. An UNC-84 mutant lacking most of its luminal domain formed functional LINC complexes, and, even though these complexes are predicted to be much shorter, the nuclear membranes of worms expressing this mutant were still spaced $50 \mathrm{~nm}$ apart. Senior author Daniel Starr thinks that inherent properties of the nuclear membranes (and the contiguous ER) might instead determine nuclear envelope spacing. Cain, N.E., et al. 2014. J. Cell Biol. http://dx.doi.org/10.1083/jcb.201405081.

\section{to a light snack}

that only lack serum and the amino acid glutamine quickly reactivate mTOR and switch off autophagy.

Chen et al. found that these "mildly starving" cells had an increased ability to take up leucine and other amino acids from the surrounding medium. The absence of glutamine activated the GAAC pathway, up-regulating amino acid transporters such as the leucine importer SLC7A5. Knocking down SLC7A5 or the GAAC transcription factor ATF4 inhibited amino acid uptake and prevented mTOR reactivation, enhancing autophagy in these cells.

The GAAC pathway therefore prevents cells from degrading their own contents when sufficient nutrients are available externally to support cell survival. Senior author Li Yu is now interested in how this pathway might operate in vivo, where it could allocate scarce resources between tissues by favoring amino acid uptake in some cell types while permitting autophagy to occur in others. Chen, R., et al. 2014. J. Cell Biol. http://dx.doi.org/10.1083/icb.201403009. lysosomes to be degraded and recycled. However, cultured cells

\section{Deubiquitination helps Rad 18 grow more tolerant}

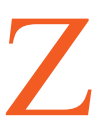

eman et al. describe how deubiquitination of the ubiquitin ligase Rad18 fine-tunes the cell's response to specific types of DNA damage.

Rad18 helps the cell's DNA replication machinery "tolerate" DNA damage and duplicate the genome with a minimal number of mutations. The ubiquitin ligase induces slightly different tolerance pathways in response to different types of DNA lesions, and it may even promote mutagenesis if activated in the absence of any damage at all. Zeman et al. therefore investigated how Rad18 is regulated.

Around a quarter of a cell's Rad18 molecules are usually ubiquitinated, probably by the enzyme itself. Rad18 was deubiquitinated, however, in response to the DNA alkylating agent MMS or $\mathrm{H}_{2} \mathrm{O}_{2}$ but not in response to other insults such as UV irradiation. MMS and $\mathrm{H}_{2} \mathrm{O}_{2}$ promote Rad18's interaction with another ubiquitin ligase, SHPRH, that is crucial for the cell's ability to tolerate these sources of DNA damage. Accordingly, ubiquitinated Rad18 didn't bind SHPRH and instead preferred to dimerize with other, nonubiquitinated Rad18 molecules, potentially sequestering them in an inactive state. Ubiquitinated $\operatorname{Rad} 18$ failed to localize to sites of DNA damage and was unable to ubiquitinate its downstream targets. Cells overexpressing a constitutively ubiquitinated form of Rad18 were less able to tolerate MMS and thus showed an increased rate of mutagenesis.

Ubiquitination therefore inhibits Rad18's activity in undamaged cells, but this posttranslational modification is quickly removed in response to MMS or $\mathrm{H}_{2} \mathrm{O}_{2}$, allowing the ubiquitin ligase to bind SHPRH and promote tolerance of the ensuing DNA damage. The authors now want to investigate how Rad18 is deubiquitinated in response to these DNA damage agents.

Zeman, M.K., et al. 2014. J. Cell Biol. http://dx.doi.org/10.1083/jcb.201311063. 